\title{
Curtición de pieles bovinas con Caelsalpinia spinosa en combinación con oxazolidina
}

César Arturo Puente Guijarro ${ }^{1}$

\begin{abstract}
RESUMEN
La curtición mineral (cromo III) es la más utilizada a nivel mundial, produce efectos nocivos al ambiente no solo en el proceso, sino también cuando los artículos han cumplido su vida útil y deben ser desechados a los vertederos de basura. En ese lugar, el contacto con agentes oxidantes puede producir la degradación del cromo trivalente a hexavalente, el cual es un compuesto altamente contaminante. El presente estudio tiene por finalidad determinar el efecto de una curtición con Caelsalpinia spinosa (tara) en combinación con oxazolidina, para crear una curtición agradable con el ambiente al reemplazar al cromo III. Como conclusión se determina que, con la utilización de oxazolidina más Caelsalpinia spinosa, se consiguen cueros con resistencias físicas elevadas que superan los estándares de calidad de las normas técnicas, así como una apreciación sensorial muy atractiva para el juez tanto en los cueros como en los artículos de marroquinería.
\end{abstract}

Palabras-claves: Curtición; cromo; tanino vegetal; oxazolidina; tara

\section{INTRODUCCIÓN}

Es conocido que una actividad ancestral efectuada por millares de familias en todo el mundo es la curtición de las pieles de animales no solo domésticos, sino salvajes, para producir cuero. Este recurso, en manos de artesanos de nuestro país, se trasforma en artículos de cuero únicos, manteniendo a la industria curtidora junto con diseñadores en la producción de artículos de cuero no sustituibles por productos sintéticos. De esta forma, el cuero como materia prima sirve para elaborar artículos para marroquinería, calzado e indumentaria, bajo las tendencias mundiales de moda y como respuesta a la gran necesidad de puestos de trabajo y competitividad extranjera. Los requerimientos ambientales que se están adoptando a nivel mundial son cada vez más exigentes; por ello, las industrias curtidoras, desde las curtiembres pequeñas de tipo artesanal hasta las de la gran industria curtidora, deben cumplir las normativas de cada país. Existe entonces una obligación de realizar cambios y adoptar nuevas tecnologías de producción que garanticen una eficaz y eficiente gestión de la industria curtidora, tomando como meta la sostenibilidad medioambiental, social y económica. Así, las curtiembres que no vayan de acuerdo al desarrollo de nuevas tecnologías limpias estarán destinadas a desaparecer, por lo que se requiere que la información deba estar a disposición de los dueños de pequeñas y medianas curtiembres (Mayta y Mayta, 2017).

El cuero, como producto final del proceso de curtición de pieles, presenta características únicas que no son sustituidas por productos sintéticos; es posible citar el porcentaje de absorción de humedad, la elasticidad y la maleabilidad que presentan, entre otras propiedades. En la presente investigación se pretende producir un cuero libre de cromo utilizando una tecnología que consiste en la obtención de pieles exentas de metales con aspecto y cualidades adecuadas. Esto será logrado mediante la sustitución de los productos curtientes habituales (sales básicas de cromo trivalente) por oxazolidina en combinación con agentes curtientes sintéticos o vegetales, empleando formulaciones semejantes a las habituales y realizando el proceso de curtición en las mismas instalaciones y equipos (Reissig, 2006). En otras palabras, lo que se busca

1 Magíster en Protección Ambiental por la Universidad Nacional Mayor de San Marcos. Actualmente, es docente de la Escuela Superior Politécnica de Chimborazo, Chimborazo, Ecuador.

E-mail: cuerosalce@yahoo.com 
es producir pieles más biodegradables, con aspecto y propiedades adecuadas para la fabricación de calzado y otros artículos de piel.

\section{METODOLOGÍA}

\section{Tipo y diseño de investigación}

Tomando en cuenta que experimentar es una modalidad de aprendizaje a través de una sistemática variación de condiciones y obtención de efectos, se va a trabajar bajo la influencia de variables en condiciones controladas para determinar los resultados al manipular las concentraciones de los nuevos curtientes. Para este caso, se trata de obtener o mantener un cuero de alta calidad (variable dependiente) después de aplicar la oxazolidina en combinación con la tara (variable independiente), realizando diferentes experimentos en el laboratorio y manipulando las cantidades de concentración de esta última variable, para luego compararlos con una curtición tradicional como es el cromo.

\section{Diseño de la investigación}

Para efectuar el ensayo del presente estudio se procedió a evaluar la calidad física, sensorial y mecánica del cuero bovino, así como su comportamiento en el artículo confeccionado (portafolios). Además, se dividió el trabajo experimental en tres fases: la primera utilizó 15 pieles; la segunda, 30 pieles; y, finalmente, la tercera consistió en la comparación de los mejores niveles tanto de la primera como de la segunda fase.

\subsection{Primera fase}

Para evaluar una curtición con diferentes niveles de sulfato de cromo $(5 \%, 6 \%$ y $7 \%)$, para la confección de artículos de marroquinería, se manipularon 15 pieles vacunas en total, distribuidas en 3 tratamientos, con 5 repeticiones por tratamiento. Los resultados experimentales de las pruebas fisicomecánicas fueron analizados bajo un Diseño Completamente al Azar (DCA), pues cumple con los principios de este tipo de proyecto. En la Tabla 1 se representa el esquema de la experimentación que fue utilizado en la investigación.

En la Tabla 2 se representa el esquema del análisis de varianza utilizado en la investigación.

\subsection{Segunda fase \\ Unidades experimentales segunda fase}

Las unidades experimentales de la curtición con diferentes niveles (12\%, 15\% y 18\%) de Caelsalpinia spinosa (tara) en combinación con $5 \%$ de oxazolidina (segunda fase), fueron 30 pieles vacunas de animales adultos adquiridas a introductores de ganado de la ciudad de Ambato.

\section{Tratamiento y diseño experimental segunda fase}

Para la segunda fase de investigación se evaluó la curtición con diferentes concentraciones de Caelsalpinia spinosa $(12 \%, 15 \%$ y $18 \%)$, mezclada con $5 \%$ de concentración de oxazolidina, las cuales fueron seleccionadas para cuero de marroquinería. Además, se utilizaron 30 pieles vacunas distribuidas en 3 concentraciones (tratamientos) con 10 repeticiones cada una. En el procesamiento de datos los resultados fueron distribuidos con un Diseño Completamente al Azar (DCA) simple. En la Tabla 3 se representa el esquema del experimento que fue utilizado en la investigación.

En la Tabla 4 se detalla el esquema del análisis de varianza utilizado en la investigación.

Tabla 1. Esquema del experimento.

\begin{tabular}{|c|c|c|c|c|}
\hline Porcentajes de cromo & Código & Repeticiones & Tamaño de la unidad experimental & Total unidades experimentales \\
\hline $5 \%$ & T1 & 5 & 1 & 5 \\
\hline $6 \%$ & T2 & 5 & 1 & 5 \\
\hline $7 \%$ & T3 & 5 & 1 & 5 \\
\hline \multicolumn{7}{|c|}{ Total de pieles } \\
\hline
\end{tabular}

Fuente: Elaboración propia.

Tabla 2. Análisis de varianza.

\begin{tabular}{|l|c|}
\hline \multicolumn{1}{|c|}{ Fuente de variación } & Grados de libertad \\
\hline Total & 14 \\
\hline Tratamiento & 2 \\
\hline Error & 12 \\
\hline
\end{tabular}

Fuente: Elaboración propia. 


\subsection{Tercera fase}

La tercera fase de la investigación fue constituida por la comparación entre los resultados del tratamiento más alto de la primera fase versus los resultados más altos de la segunda fase. Asimismo, para su tabulación se utilizó una estadística descriptiva, en la cual se utilizaron medidas de tendencia central, como la media, la mediana y la moda. Además, medidas de dispersión, como la varianza y la desviación estándar. Finalmente, se utilizó la prueba de contraste t de Student para determinar si existen o no diferencias.

\section{Técnicas de análisis de datos}

Los resultados físicos, mecánicos y sensoriales del cuero y producto confeccionado se tabularon utilizando el programa estadístico Infostat (versión 10) y la hoja de cálculo estadístico Excel 2013. Por otro lado, para el caso de las estadísticas descriptivas, también se utilizarán gráficos para visualizar los resultados.

\section{RESULTADOS Y DISCUSIÓN}

\section{Primera fase}

1.1. Valoración de las calificaciones físicas de los cueros vacunos curtidos con diferentes niveles $(5 \%, 6 \%$ y $7 \%)$ de cromo

\subsubsection{Resistencia a la tensión}

Los resultados de la resistencia a la tensión de los cueros vacunos determinaron diferencias altamente significativas $(P<0,05)$ por efecto de la curtición con diferentes niveles de cromo. A partir de ello se establecieron los resultados, los cuales fueron más altos con $7 \%$ de cromo (T3), alcanzando los
$2057,37 \mathrm{~N} / \mathrm{cm}^{2}$; mientras que descendieron al utilizar $6 \%$ de cromo (T2), llegando a los 1887,53 N/ $\mathrm{cm}^{2}$. En otras palabras, los resultados más altos se obtuvieron al utilizar mayores niveles de cromo, ya que se consiguió el fortalecimiento del entretejido colagénico, de tal manera que las fibras de colágeno soporten fuerzas multidireccionales a las que son sometidas en el momento del armado o del uso diario que impiden su ruptura prematura y, con ello, la vida útil del cuero disminuya así como su calidad y precio (Silvateam, s. f.).

\subsubsection{Porcentaje de elongación}

Los resultados del porcentaje de elongación de los cueros vacunos determinaron diferencias altamente significativas por efecto de la curtición con diferentes concentraciones de cromo. Asimismo, se establecieron las mejores respuestas en el tratamiento T3 $(7 \%)$ con $94,38 \%$, seguido de los valores alcanzados en el lote de cueros del tratamiento T2 $(6 \%)$ con elongaciones de $80,50 \%$. Mientras que los resultados más bajos fueron reportados por los cueros del tratamiento T1 (5\%), alcanzando el $56,90 \%$. Es decir, al curtir con mayores niveles de cromo se mejoró la elasticidad del cuero, lo que, según INESCOP (2011), hizo lograr un alargamiento adecuado para que se traslade fácilmente de la forma espacial a la multidireccional al adoptar la estructura del artículo que se confecciona.

\subsubsection{Resistencia al desgarro}

La evaluación de la resistencia al desgarro determinó los resultados más elevados en los cueros del tratamiento T3 $(7 \%)$, obteniendo $55,85 \mathrm{~N}$, seguido de los valores alcanzados por los cueros del tratamiento $\mathrm{T} 1(5 \%)$, que registraron promedios de

Tabla 3. Esquema del experimento.

\begin{tabular}{|c|c|c|c|c|}
\hline $\begin{array}{c}\text { Concentraciones de } \\
\text { Caelsalpinia spinosa }\end{array}$ & Código & Repeticiones & Tamaño de la unidad experimental & Total unidades experimentales \\
\hline $12 \%$ & T1 & 10 & 1 & 10 \\
\hline $15 \%$ & T2 & 10 & 1 & 10 \\
\hline $18 \%$ & T3 & 10 & 1 & 10 \\
\hline \multicolumn{7}{|c|}{ Total de pieles } \\
\hline
\end{tabular}

Fuente: Elaboración propia.

Tabla 4. Esquema del ADEVA.

\begin{tabular}{|l|c|}
\hline Fuente de variación & Grados de libertad \\
\hline Total & 29 \\
\hline Tratamiento & 2 \\
\hline Error & 27 \\
\hline
\end{tabular}

Fuente: Elaboración propia. 
$54,35 \mathrm{~N}$. Asimismo, los resultados más bajos fueron determinados en el tratamiento T2 $(7 \%)$, llegando a valores de 53,67 N, como se indica en la Tabla 5.

\subsection{Evaluación de las calificaciones sensoriales del cuero vacuno curtido con diferentes concentraciones de cromo}

\subsubsection{Llenura}

Los valores de llenura de los cueros vacunos registraron diferencias estadísticas. Según la prueba de Kruskal-Wallis, se estableció que los resultados más altos se dan al utilizar en la curtición $7 \%$ de cromo (T3), ya que las respuestas son de 4,80 puntos, considerado por Hidalgo (2017) como una calificación excelente. Por otro lado, las respuestas bajas fueron determinadas en los cueros obtenidos con niveles bajos de cromo, es decir, con 5\% (T1), pues las ponderaciones fueron de 3,20 puntos.

\subsubsection{Finura de flor}

El análisis de la finura de flor determinó diferencias altamente significativas $(P<0,01)$ por efecto de la inclusión de diferentes concentraciones de cromo. Además, se establecieron los resultados más altos en los cueros del tratamiento T3 $(7 \%)$, alcanzando los 4,60 puntos, lo cual Hidalgo (2017) considera una calificación excelente. A continuación, se ubicaron los registros alcanzados en los cueros del tratamiento T2 (6\%), cuyos valores fueron de 4,20 puntos, la cual es una calificación muy buena de acuerdo con Hidalgo (2017). Finalmente, en la separación de medias, a través de la prueba de Tukey (ANOVA), se reportó la finura de flor expuesta en el tratamiento T1 (5\%), logrando resultados de 2,80 puntos y calificación baja.

\subsubsection{Redondez}

La redondez presentó diferencias altamente significativas $(P<0,01)$. Así, se establecieron las mejores respuestas en los cueros del tratamiento T3 $(7 \%)$, obteniendo valores de 4,80 puntos y una calificación excelente por Hidalgo (2017). Luego, en forma descendente, la redondez reportada en los cueros del tratamiento T2 (6\%) alcanzó los valores de 4,20 puntos y ponderación de muy buena. Por el contrario, los resultados más bajos fueron registrados en el tratamiento T1 (5\%), logrando respuestas de 3,0 puntos y calificación buena.

\section{Segunda fase}

2.1. Valoración de las resistencias físicas de los cueros vacunos curtidos con $5 \%$ de oxazolidina más diferentes niveles $(12 \%, 15 \%$ y $18 \%)$ de tara

\subsubsection{Resistencia a la tracción}

Los valores de la resistencia a la tracción no determinaron estadísticamente diferencias $(P<0,05)$ por efecto de la inclusión de diferentes niveles de Caelsalpinia spinoza (tara), en combinación con $5 \%$ de oxazolidina. De esta forma, se establecieron los resultados más altos en el tratamiento T2 (15\%), obteniendo $2855,15 \mathrm{~N} / \mathrm{cm} 2$, seguido de los reportes del tratamiento T3 (18\%), logrando respuestas de $2717,64 \mathrm{~N} / \mathrm{cm} 2$. Por otro lado, los valores más bajos fueron reportados en los cueros del tratamiento T1 $(12 \%)$, alcanzando medias de tracción de $2501,54 \mathrm{~N} / \mathrm{cm}^{2}$.

\subsubsection{Porcentaje de elongación}

El porcentaje de elongación en el análisis de varianza no determinó diferencias estadísticas $(P>0,05)$ entre las medias de los tratamientos por efecto de la inclusión de diferentes niveles de tara en combinación con oxazolidina. De este modo, se estableció el mayor porcentaje de elongación en el tratamiento T3 (18\%), logrando respuestas de $61,54 \%$, las cuales descendieron a $59,55 \%$ en los cueros del tratamiento T1 $(12 \%)$. Por el contrario, los resultados más bajos de elongación fueron reportados por el tratamiento T2 (15\%), obteniendo respuestas de $56,93 \%$.

\subsubsection{Resistencia al desgarro}

La evaluación de la resistencia al desgarro no determinó diferencias estadísticas $(P>0,05)$ entre tratamientos por efecto de la aplicación de diferentes niveles de tara en combinación con oxazolidina.

Tabla 5. Evaluación de las valoraciones físicas de los cueros vacunos curtidos con diferentes niveles (5\%, 6\% y 7\%) de cromo (primera fase).

\begin{tabular}{|c|c|c|c|c|c|c|c|c|c|c|}
\hline \multirow[b]{2}{*}{ Características físicas } & \multicolumn{6}{|c|}{ Niveles de cromo } & \multirow{3}{*}{$\begin{array}{c}\text { EE (Error } \\
\text { Estándar) } \\
129,46\end{array}$} & \multirow{3}{*}{$\begin{array}{l}\text { Prob. } \\
0,0142 \\
\end{array}$} & \multirow{3}{*}{$\begin{array}{l}\text { Sign. } \\
\text { NS }\end{array}$} & \multirow{3}{*}{$\begin{array}{c}\text { CV (Coeficiente } \\
\text { de Variación) }\end{array}$} \\
\hline & \multicolumn{2}{|l|}{$\begin{array}{l}5 \% \\
\text { T1 }\end{array}$} & \multicolumn{2}{|c|}{$\begin{array}{l}6 \% \\
\text { T2 } \\
\end{array}$} & \multicolumn{2}{|l|}{$\begin{array}{l}7 \% \\
\text { T3 }\end{array}$} & & & & \\
\hline Resistencia a la tensión $\left(\mathrm{N} / \mathrm{cm}^{2}\right)$ & 1341,12 & a & 1887,53 & a & 2057,37 & a & & & & \\
\hline Porcentaje de elongación (\%) & 56,90 & a & 80,50 & a & 94,38 & a & 6,78 & 0,0056 & ** & 19,62 \\
\hline Resistencia al desgarro (N) & 54,35 & a & 53,67 & a & 55,85 & a & 1,32 & 0,157 & NS & 5,4 \\
\hline
\end{tabular}

Fuente: Elaboración propia. 
Así, se establecieron las respuestas más altas en el tratamiento T3 (18\%), obteniendo 117,78 N (ver Tabla 6), es decir, a mayores niveles de tara combinada con oxazolidina, se consiguió una mayor resistencia al desgarro de los cueros vacunos. En ese sentido, Vargas y Amurrio (2017) indican que al frotar las superficies sea entre sí o con cuerpos extraños no se producen daños, ni en la superficie de la piel, ni en su estructura interna, lo cual implica que se trata de un cuero muy rígido que al mínimo estiramiento se romperá. Por lo tanto, se considera que al alcanzarse una mayor resistencia del cuero a rasguños con su propia superficie o con cuerpos extraños que debilitan el entretejido fibrilar, se provocan fisuras que terminan en un envejecimiento prematuro (Casa Química Bayer, 2007).

2.2. Valoración de las calificaciones sensoriales de los cueros vacunos curtidos con $5 \%$ de oxazolidina más diferentes niveles $(12 \%, 15 \%$ y $18 \%$ ) de tara

\subsubsection{Llenura}

La valoración de llenura determinó diferencias altamente significativas $(P<0,01)$ según la prueba de Kruskal-Wallis, pues se establecieron las respuestas más altas en el tratamiento T3 (18\%), obteniendo 4,80 puntos y condición excelente.

\subsubsection{Finura de flor}

La evaluación de la finura de flor determinó diferencias altamente significativas $(P<0,01)$ por efecto de la inclusión a la fórmula del curtido de diferentes niveles de tara más $5 \%$ de oxazolidina. De esta forma, se determinaron las respuestas más altas al utilizar $18 \%$ de tara (T3), alcanzando respuestas de 4,70 puntos (ver Tabla 7) y calificación excelente según Hidalgo (2017).

\subsubsection{Redondez}

El análisis de la redondez reportó diferencias altamente significativas $(P<0,01)$, según el criterio de la prueba de Kruskal-Wallis, por efecto de la utilización de diferentes niveles de tara en combinación con 5\% de oxazolidina. Así, se establecieron las respuestas más altas al utilizar $12 \%$ de tara (T1), ya que los resultados fueron de 4,70 puntos y calificación excelente según Hidalgo (2017). A continuación, se apreciaron los resultados alcanzados en el lote de cueros curtidos con $15 \%$ de tara (T2), pues las respuestas fueron de 3,90 puntos y calificación muy buena. Por otro lado, la redondez más baja se apreció en los cueros curtidos con $12 \%$ de tara con ponderaciones de 3,40 puntos y condición buena.

\subsubsection{Plenitud}

Las medias obtenidas de los resultados de la plenitud de los cueros vacunos registraron diferencias

Tabla 6. Evaluación de las resistencias físicas de los cueros vacunos curtidos con $5 \%$ de oxazolidina más diferentes niveles (12\%, 15\% y 18\%) de tara.

\begin{tabular}{|c|c|c|c|c|c|c|c|}
\hline \multirow{2}{*}{ Resistencias físicas } & \multicolumn{3}{|c|}{$\begin{array}{l}\text { Niveles de tara más } 5 \% \text { de } \\
\text { oxazolidina }\end{array}$} & \multirow{2}{*}{$\begin{array}{l}\text { CV (Coeficiente de } \\
\text { Variación) }\end{array}$} & \multirow{2}{*}{$\begin{array}{l}\text { EE (Error } \\
\text { Estándar) }\end{array}$} & \multirow{2}{*}{ Sign. } & \multirow[t]{2}{*}{ Prob. } \\
\hline & $12 \%$ & $15 \%$ & $18 \%$ & & & & \\
\hline Resistencia a la tracción $\left(\mathrm{N} / \mathrm{cm}^{2}\right)$ & $2501,54 \mathrm{a}$ & 2855,15 a & $2717,64 \mathrm{a}$ & 25,52 & 217,21 & 0,52 & NS \\
\hline Porcentaje de elongación (\%) & 59,55 a & 56,93 a & 61,54 a & 12,58 & 2,36 & 0,40 & NS \\
\hline Resistencia al desgarro $(\mathrm{N})$ & $101,23 \mathrm{a}$ & 101,36 a & 117,78 a & 19,24 & 6,50 & 0,14 & NS \\
\hline
\end{tabular}

Fuente: Elaboración propia.

Tabla 7. Valores de las calificaciones sensoriales de los cueros vacunos curtidos con $5 \%$ de oxazolidina más diferentes niveles $(12 \%, 15 \%$ y $18 \%)$ de tara.

\begin{tabular}{|c|c|c|c|c|c|c|c|}
\hline \multirow{2}{*}{ Calificaciones sensoriales } & \multicolumn{3}{|c|}{$\begin{array}{c}\text { Niveles de tara más } 5 \% \text { de } \\
\text { oxazolidina }\end{array}$} & \multirow{2}{*}{$\begin{array}{l}\text { CV (Coeficiente de } \\
\text { Variación) }\end{array}$} & \multirow{2}{*}{$\begin{array}{l}\text { EE (Error } \\
\text { Estándar) }\end{array}$} & \multirow{2}{*}{ Sign. } & \multirow{2}{*}{ Prob. } \\
\hline & $\begin{array}{c}12 \% \\
\mathrm{~T} 1\end{array}$ & $\begin{array}{c}15 \% \\
\mathrm{~T} 2 \\
\end{array}$ & \begin{tabular}{|c|}
$18 \%$ \\
T3 \\
\end{tabular} & & & & \\
\hline Puntos de llenura & $3,10 \mathrm{c}$ & $3,80 \mathrm{~b}$ & $4,80 \mathrm{a}$ & 19,80 & 0,24 & 0,00 & ** \\
\hline Puntos de finura de flor & $3,50 \mathrm{c}$ & $4,10 \mathrm{~b}$ & $4,70 \mathrm{a}$ & 14,47 & 0,19 & 0,00 & ** \\
\hline Puntos de redondez & $4,70 \mathrm{a}$ & $3,90 \mathrm{~b}$ & $3,40 \mathrm{~b}$ & 16,24 & 0,21 & 0,00 & ** \\
\hline Puntos de plenitud & $4,50 \mathrm{a}$ & $3,60 \mathrm{~b}$ & $3,20 \mathrm{~b}$ & 14,90 & 0,18 & 0,00 & ** \\
\hline
\end{tabular}

Fuente. Elaboración propia. 
altamente significativas $(P<0,01)$ por efecto de la aplicación de diferentes niveles de tara en combinación con oxazolidina, aplicado a la formulación del curtido, se evidenció una optimización de la plenitud ante niveles más bajos de tara. De este modo, se registró en el tratamiento T1 (12\%) las puntuaciones más altas, cuya media fue de 4,50 puntos, con una ponderación de excelente entre la escala propuesta por Hidalgo (2017), y que descendió en las respuestas de los cueros del tratamiento T2 $(15 \%)$ a una media de 3,60 puntos y ponderación de muy buena.

\section{Tercera fase}

3.1. Comparación de las resistencias físicas de los cueros vacunos curtidos con $7 \%$ de cromo versus la curtición con $18 \%$ de tara en combinación con $5 \%$ de oxazolidina

\subsubsection{Resistencia a la tracción}

La evaluación de la resistencia a la tracción no determinó diferencias estadísticas $(P>0,05)$ al comparar la curtición con cromo versus diferentes niveles de tara más oxazolidina. De esta forma, se reportaron valores más altos al utilizar la curtición con tara (T2), obteniendo valores de $2214,49 \mathrm{~N} / \mathrm{cm}^{2}$ en comparación con la curtición con cromo (T1), logrando respuestas de $2057,37 \mathrm{~N} / \mathrm{cm}^{2}$. Además, el error típico fue de 55,62 y 201,98 para los tratamientos T1 (cromo) y T2 (tara), respectivamente. Los valores de las medianas registraron tensiones de 2000,00 y $2177,07 \mathrm{~N} / \mathrm{cm}^{2}$, incluso se apreció que no existió ningún valor repetido, es decir, no hubo moda; sin embargo, la desviación estándar fue alta, alcanzando cada uno de ellos resultados de $124,36 \mathrm{~N} / \mathrm{cm}^{2}$ y $451,63 \mathrm{~N} / \mathrm{cm}^{2}$.

\subsubsection{Porcentaje de elongación}

Los resultados del porcentaje de elongación de los cueros bovinos determinaron diferencias altamente significativas $(P>0,05)$. Así, se establecieron resultados más altos en el tratamiento T1(tara), ya que las respuestas fueron de $94,38 \%$ y la mediana de $80.50, \%$. Por otro lado, en los cueros del tratamiento T1(cromo) las respuestas fueron de $94.38 \%$ y $56.90 \%$, siendo necesario resaltar que los resultados en el tratamiento T2 es de $80.50 \%$.

\subsubsection{Resistencia al desgarro}

La evaluación de la resistencia al desgarro de los cueros vacunos reportó diferencias altamente significativas. De este modo, se establecieron los resultados más altos en el tratamiento T3 (tara), obteniendo $117.78 \mathrm{~N}$ y una mediana de 101,36 N, mientras que al utilizar una curtición con cromo los resultados medios fueron de $55,85 \mathrm{~N}$ y la mediana de 54,35 N. Según Chávez (2010), es necesario combinar la curtición junto a oxazolidina y recurtientes vegetales para alcanzar mayores temperaturas de contracción y obtener pieles de calidad comparable a las curtidas con cromo.

\subsection{Valores de las calificaciones sensoriales de las pieles vacunas curtidas con cromo versus la curtición con diferentes concentraciones de tara en combinación con $5 \%$ de oxazolidina}

\subsubsection{Llenura}

Los valores de llenura de los cueros vacunos reportaron diferencias estadísticas $(P<0,05)$. Así, se registraron respuestas más efectivas al utilizar la curtición con tara (T2), ya que los resultados fueron de 4,80 puntos en comparación con los cueros curtidos al vegetal que lograron 3,80 puntos, y cuyas calificaciones son de excelente y muy buena, respectivamente, según Hidalgo (2017). Lo cual es corroborado, de acuerdo con Hoinacki (2009), a través del efecto negativo que tiene la curtición con sales de cromo, sobre todo, por la trasformación de cromo trivalente a hexavalente.

\subsubsection{Redondez}

La calificación de la redondez del cuero vacuno no reportó diferencias estadísticas por efecto de la comparación de la curtición tradicional (cromo) con la vegetal (tara). De esta forma, se establecieron respuestas más altas en el tratamiento T2 (tara), puesto que sus valores medios fueron de 4,80 puntos y condición excelente, en comparación con la curtición con cromo, que registró valores de 3,60 puntos y condición muy buena. En otras palabras, los resultados en las dos curticiones son similares; sin embargo, desde el punto de vista ambiental, la mejor alternativa es trabajar con curtientes vegetales en combinación con oxazolidina, por su bajo poder contaminante (Córdova et al., 2013).

\subsubsection{Finura de flor}

Los resultados reportados por la finura de flor de los cueros vacunos determinaron diferencias estadísticas $(P>0,05)$. Así, se estableció un valor promedio de 4,80 y 3,40 puntos para el tratamiento T1 (cromo) y T2 (tara), respectivamente, y calificaciones que fueron de excelente a buena, es decir, que al cutir con cromo se aprecian mejores respuestas de finura de flor. En ese sentido, Cotance (2004) señala que la finura de flor se debe a que la combinación del grupo carboxílico del colágeno de una fibra con el cromo es muy alta, la cual forma un enlace bastante fuerte que atrae a la fibra adyacente, lo que 
da como resultado que el folículo piloso se cierre, dando lisura a la superficie de la flor.

Por otro lado, Bacardit (2005) menciona que la curtición mantiene las propiedades más deseadas de la piel, es decir, la resistencia al desgaste, a la humedad y a la flexibilidad. Además, su aspecto exterior agradable al tacto y a la vista es logrado por los diferentes tipos de enlace con el colágeno retícula sin cambiar la estructura de las fibras naturales, permitiendo que el material esté disponible en diferentes espacios y tiempos de tal manera que se pueda satisfacer con la demanda del consumidor.

\section{Comportamiento del cuero en la confección de artículos de marroquinería}

\subsection{Pespunte}

La valoración sensorial de los artículos confeccionados con cuero curtido con diferentes niveles de Caelsalpinia spinosa (tara) determinó entre las medias diferencias altamente significativas que las mayores puntuaciones se lograron al elaborar los portafolios con cuero del tratamiento T3 (18\%), ya que la calificación alcanzada fue de 4,80 puntos y condición excelente, según la ponderación de Santillan (2017). Además, se redujo a 4,30 puntos en los artículos confeccionados con cueros del tratamiento T2 (15\%), donde la ponderación fue de 4,30 puntos y condición muy buena. Por el contrario, las puntuaciones más bajas fueron registradas en los artículos del tratamiento T1 (12\%), logrando una puntuación de 3,60 puntos y una calificación buena. Asimismo, Soler (2004) indica que el pespunte es el ensamble de las piezas del corte mediante operaciones de costura, pegado, colocación de adornos y herrajes, etc., y que el cuero debe presentar una resistencia adecuada para soportar esta etapa del proceso industrial, que muchas veces es fuerte, puesto que se debe evitar arrugas o deformaciones que desmejoran la presentación del calzado.

\subsection{Corte}

La variable corte del cuero determinó diferencias altamente significativas por efecto de la curtición con diferentes concentraciones de Caelsalpinia spinosa más $5 \%$ de oxazolidina. Así, se establecieron las calificaciones más altas al utilizar mayores niveles de tara (T3), ya que la calificación, declarada por el juez, determinó puntuaciones de 4,90 puntos y condición excelente, que descendieron a 4,30 puntos y condición muy buena. Por otra parte, las respuestas más bajas fueron registradas en el tratamiento T1 $(12 \%)$, ya que la calificación fue de 3,90 puntos y condición buena.

\section{RECOMENDACIONES}

- Al curtir las pieles bovinas con oxazolidina y Caelsalpinia spinosa se consigue elevar su clasificación, disminuir los defectos, mejorar las propiedades físicas, sensoriales y conseguir una elevada estabilidad del cuero frente a los procesos de fabricación y el paso del tiempo, eliminando la presencia de cromo III.

- La proporción de oxazolidina empleada (5\%) en combinación con $12 \%$ de Caelsalpinia spinosa mejora el aspecto y las propiedades físicas del cuero vacuno. También se alcanza una temperatura de contracción de $80^{\circ} \mathrm{C}$, lo que además economiza el proceso, donde los réditos económicos son mayores y superan ampliamente las ganancias generadas al curtir con cromo.

- La utilización de la oxazolidina como agente curtiente en combinación con Caelsalpinia spinosa permite obtener pieles de calidad que pueden ser utilizadas por las industrias de marroquinería. Así lo demuestran los resultados de las pruebas físicas y sensoriales y, sobre todo, la calificación de los artículos confeccionados.

- Al curtir con oxazolidina en combinación con Caelsalpinia spinosa, se encuentra un pequeño incremento de la Demanda Biológica de Oxígeno (DBO) y la Demanda Química de Oxígeno (DQO), el cual no cumple con las normativas del Texto Unificado de Legislación Secundaria (TULSMA). Al realizar el tratamiento se consiguió que este valor fuera menor al límite permisible.

- La evaluación del comportamiento del cuero, frente a las condiciones de confección de marroquinería más representativas, determinó que los mejores resultados de pespunte y corte del cuero se aprecian al utilizar $18 \%$ de tara en combinación con $5 \%$ de oxazolidina (T3), ya que las calificaciones van de 4,80 puntos y 4,90 puntos, respectivamente y condición excelente.

\section{CONCLUSIONES}

- Aplicar la combinación de Caelsalpinia spinosa (tara) con oxazolidina, como un sustituto ecológico del cromo en el proceso de curtición de pieles bovinas, va de acuerdo a la legislación ambiental, pues el uso del cromo en la curtición está prohibido por los 
efectos negativos que ocasiona al ambiente al transformarse en cromo hexavalente, incluso después de ser desechado el artículo final (Hoinacki, 2009).

- Utilizar la combinación de $12 \%$ de Caelsalpinia spinosa (tara) más el $5 \%$ de oxazolidina para fabricar cueros que cumplen los estándares de calidad en las pruebas físicas y sensoriales, el cual está recomendado para la fabricación de diferentes artículos de piel, así como bajo los criterios establecidos en la obtención de la ecoetiqueta europea del cuero.

- Investigar la utilización de la combinación de Caelsalpinia spinosa más oxazolidina en otro tipo de pieles como ovinas, caprinas o especies menores como aves, pescado, conejos, entre otras, para validar la tecnología creada y de esa manera suplir la necesidad de materia prima que tiene un costo más bajo (Chávez, 2010).

\section{REFERENCIAS BIBLIOGRÁFICAS}

[1] Bacardit, A. (2005). Química técnica del cuero. Cataluña, España: COUSO.

[2] Silvateam (s. f.). Cuero curtido al vegetal. Silvateam. Recuperado de https://www. silvateam.com/es/quienes-somos/extraidosde-la-naturaleza/cuero-curtido-al-vegetal.html

[3] Casa Química Bayer (2007). Resistencia al frote del acabado del cuero. Barcelona, España: IMANAL.

[4] Chávez, A. (2010). Descripción de la nocividad del cromo proveniente de la industria curtiembre y de las posibles formas de removerlo. Revista Ingenierías, 9(17), 4149. Recuperado de https://dialnet.unirioja.es/ descarga/articulo/4845697.pdf

[5] Córdova, H., Vargas, R., Téllez, L., Cesare, M., Becker, R. y Visitación, L. (2013). Influencia del uso de acomplejantes en el baño de curtido sobre la calidad final del cuero. Revista de la Sociedad Química, 79(4), 388-397.

[6] Cotance, A. (2004). Ciencia y Tecnología en la Industria del Cuero. Igualada, España: Curtidores Europeos.

[7] Hidalgo, L. (2017). Escala de calificación de las variables sensoriales del cuero bovino curtido con diferentes niveles de tara. Riobamba, Ecuador: ESPOCH.

[8] Hoinacki, E. (2009). Peles e couros. Origens, defeitos e industrialização. Porto Alegre, Brasil: SENAI/RS.

[9] INESCOP (2011). Piel Respetuosa con el Medio Ambiente Curtida con Oxazolidina. Alicante, España: INESCOP.

[10] Patudo, A. (2012). Qué es hipótesis y cómo se estructura. Recuperado de https://es.scribd. com/document/105144657/Que-es-hipotesisy-como-se-estructura

[11] Reissig, P. (2006). Innovación en Cuero = Oportunidad para el Diseño. Recuperado de https://www.inti.gob.ar/disenoindustrial/pdf/ reissig.pdf

[12] Mayta, R. y Mayta, J. (2017). Remoción de cromo y demanda química de oxígeno de aguas residuales de curtiembre por electrocoagulación. Revista de la Sociedad Química, 83(3), 331-340.

[13] Santillán, A. (2017). Escala de apreciación de las características funcionales del cuero bovino. Riobamba, Ecuador: El Al-CE.

[14] Soler, J. (2004). Procesos de curtido. Barcelona, España: CETI.

[15] Vargas, D. y Amurrio, D. (2017). Alternativa de proceso de curtido con alto agotamiento de Cromo para las curtiembres tradicionales de la ciudad de Cochabamba. Acta Nova, 8(1), 3-30. 


\section{Cowhide Tanning using Caelsalpinia spinosa combined with Oxazolidine}

CÉsar Arturo Puente Guijarro ${ }^{1}$

\begin{abstract}
Mineral tanning (chromium III) is the most used tanning method worldwide, causing harmful environmental effects not only during production, but also after the goods have completed their lifespan and are discarded into landfills. There, contact with oxidizing agents may result in the degradation of trivalent chromium into hexavalent chromium, a highly contaminating compound. This study attempts to determine the effect of tanning using Caelsalpinia spinosa (tara) combined with oxazolidine, to create an environmentally friendly tanning method by replacing chromium III. In conclusion, it is determined that the use of Caelsalpinia Spinosa combined with oxazolidine produces tanned hides with high physical strength properties that exceed technical regulatory quality standards, as well as a sensory appreciation that is attractive for the user of both tanned hides and leather goods.
\end{abstract}

Keywords: Tanning; chrome; vegetable tannin; oxazolidine; tara.

\section{INTRODUCTION}

It is well known that hide tanning, of both domestic and wild animals, to produce leather is an ancestral activity performed by thousands of families worldwide. In the hands of artisans from our country, this resource is transformed into unique leather goods, therefore, supporting the tanning industry and designers of leather goods that cannot be replaced by synthetic items. Thus, leather as raw material is used to produce leather accessories, footwear and apparel in line with international trends, responding to the need for employment and international competitiveness. Environmental regulations that are being adopted worldwide are increasingly more demanding; as a result, tanning enterprises, from small-scale artisanal tanneries to large-scale tanneries, must comply with national regulations. Therefore, an obligation exists to make changes and adopt new production technologies that guarantee the effective and efficient management of the tanning industry, in order to achieve environmental, social and economic sustainability goals. Tanneries that fail to comply with the implementation of new clean technologies are destined to disappear, thus, information should be available to the owners of small and medium size tanneries. (Mayta \& Mayta, 2017).

Leather, as the final product of the hide tanning process, has unique characteristics that cannot be replaced by synthetic products such as the percentage of humidity absorption, elasticity, and malleability, among other properties. This study attempts to produce a chrome-free leather using technology that enables the production of metal-free leather with adecuate appearance and properties. This is achieved by replacing the usual tanning agents (simple trivalent chromium salts) with oxazolidine combined with synthetic or vegetable tanning agents, using formulations similar to those usually used and conducting the tanning process in the same facilities using the same equipement (Reissig, 2006). In other words, the objective is to produce more biodegradable leathers that maintain the appearance and properties required for leather footwear and other goods.

1 Master's in Environmental Protection from the Universidad Nacional Mayor de San Marcos Currently working as Professor at the Escuela Superior Politécnica de Chimborazo, Chimborazo, Ecuador.

E-mail: cuerosalce@yahoo.com 


\section{METHODOLOGY}

\section{Research type and design}

Considering that experimentation is a form of learning through systematic variation of conditions and achieved results, this research studies two variables under controlled conditions to determine results obtained after manipulating the concentrations of the new tanning agents. In this case, the objective is to achieve or maintain a good quality leather (dependent variable) after the application of oxazolidine combined with tara (independent variable), conducting several laboratory experiments and manipulating the concentration amounts of the independent variable, to be later compared with a traditional chrome tanning method.

\section{Research design}

Physical, sensory and mechanical qualities of cowhide leather were evaluated in this study, as well as its behavior after it had been transformed into a manufactured item (briefcase). In addition, the experimental work was divided into three stages: the first stage used 15 hides; the second, 30 hides; and the third stage consisted on comparing the highest values obtained in both, the first and second stage.

\subsection{First stage}

To evaluate a tanning method using different chromium sulfate concentrations $(5 \%, 6 \%$ and $7 \%)$ for the manufacture of leather goods, a total of 15 cowhides were prepared, distributed in three groups subjected to three different treatments with five repetitions per treatment. The findings of the physico-mechanical experiments were analyzed using a completely randomized design (CRD), which satisfies the criteria of this type of project. Table 1 shows the experiment scheme used in the study.

Table 2 shows the scheme of the analysis of variance used in the study.

\subsection{Second stage}

\section{Experimental units - Second stage}

The experimental units used for tanning using different concentrations of Caelsalpinia spinosa (tara) $(12 \%, 15 \%$ and $18 \%)$ combined with $5 \%$ oxazolidine (second stage) were 30 cowhides of adult animals purchased from cattle dealers from the city of Ambato.

\section{Treatment and experimental design - Second stage}

For the second stage of the study, tanning using different concentrations of Caelsalpinia spinose $(12 \%$, $15 \%$ and $18 \%$ ) combined with $5 \%$ oxazolidine was evaluated. A total of 30 cowhides, selected for leather goods, distributed in three groups exposed to different concentrations (treatments) with 10 repetitions per treatment were used. The findings were distributed using a completely randomized design (CRD) during the data processing. Table 3 shows the experiment scheme used in the study.

Table 4 shows the scheme of the analysis of variance used in the research.

\subsection{Third stage}

The third stage of the study consisted of comparing the highest values obtained in the treatments of the first and second stage. A descriptive statistic, including measures of central tendency such as

Table 1. Experiment scheme.

\begin{tabular}{|c|c|c|c|c|}
\hline Percentage of chromium & Code & Repetitions & Size of experimental unit & Total of experimental units \\
\hline $5 \%$ & T1 & 5 & 1 & 5 \\
\hline $6 \%$ & T2 & 5 & 1 & 5 \\
\hline $7 \%$ & T3 & 5 & 1 & 5 \\
\hline \multicolumn{7}{r}{ Total number of hides } \\
\hline
\end{tabular}

Source: Prepared by the author.

Table 2. Analysis of variance.

\begin{tabular}{|l|c|}
\hline Source of variation & Degrees of freedom \\
\hline Total & 14 \\
\hline Treatment & 2 \\
\hline Error & 12 \\
\hline
\end{tabular}

Source: Prepared by the author. 
media, median and mode, was used to tabulate the results. Measures of statistical dispersion such as variance and standard deviation were used as well. Finally, Student's comparison t-test was used to determine whether or not there were differences.

\section{Data analysis techniques}

The physical, mechanical and sensory results of leather and manufactured product were tabulated by means of the statistical software InfoStat (version 10) and statistical spreadsheet Excel 2013. Conversely, charts were used to visualize the results for descriptive statistics.

\section{RESULTS AND DISCUSSION}

\section{First stage}

1.1. Evaluation of the physical properties of cowhides tanned using different concentrations of chromium $(5 \%, 6 \%$ and $7 \%)$

\subsubsection{Tensile strength}

Highly significant differences were determined for tensile strength of the cowhides subjected to tanning using different concentrations of chromium.

Highly significant differences $(P<0.05)$ regarding tensile strength of cowhides were determined, as a result of tanning using different concentrations of chromium. The highest values were obtained from tanning using 7\% chromium concentration (T3), producing results of $2057.37 \mathrm{~N} / \mathrm{cm}^{2}$; whereas values decreased when using $6 \%$ chromium concentration (T2), producing results of $1887.53 \mathrm{~N} / \mathrm{cm}^{2}$. In other words, the highest results were obtained using higher concentrations of chromium, because interwoven collagen fibers were strengthened enabling them to withstand multidirectional forces to which these fibers are subjected during the assembly process or daily use, thus preventing premature rupture of leather as well as preventing its useful life of leather from decreasing, along with its quality and price (Silvateam, n.d.).

\subsubsection{Percentage of elongation}

Highly significant differences regarding elongation percentage of cowhide leathers were determined, as a result of tanning with different concentrations of chromium. Likewise, the best results were obtained by the group of hides subjected to treatment T3 $(7 \%)$ with elongation values of $94.38 \%$, followed by the group subjected to treatment T2 (6\%) with elongation values of $80.50 \%$; whereas the lowest results were obtained by the group subjected to treatment T1 (5\%), with values of $56.90 \%$. This is to say tanning using higher chromium concentrations enhanced leather elasticity, which, according to INESCOP (2011), made it possible to achieve adequate stretching that transfers easily into multidirectional shapes when conforming to the shape of the object being produced.

\subsubsection{Tear strength}

The highest values regarding tear strength were determined in the group of hides subjected to treatment T3 $(7 \%)$, obtaining $55.85 \mathrm{~N}$, followed by the group of hides subjected to treatment T1 (5\%), achieving values of $54.35 \mathrm{~N}$; whereas the lowest results were obtained by the group of hides subjected to treatment T2 (7\%), with values of $53.67 \mathrm{~N}$, as shown in Table 5.

Table 3. Experiment scheme.

\begin{tabular}{|c|c|c|c|c|}
\hline Percentage of chromium & Code & Repetitions & Size of experimental unit & Total of experimental units \\
\hline $12 \%$ & T1 & 10 & 1 & 10 \\
\hline $15 \%$ & T2 & 10 & 1 & 10 \\
\hline $18 \%$ & T3 & 10 & 1 & 10 \\
\hline \multicolumn{7}{|c|}{ Total number of hides } \\
\hline
\end{tabular}

Source: Prepared by the author.

Table 4. ANOVA scheme.

\begin{tabular}{|l|c|}
\hline Source of variation & Degrees of freedom \\
\hline Total & 29 \\
\hline Treatment & 2 \\
\hline Error & 27 \\
\hline
\end{tabular}

Source: Prepared by the author. 


\subsection{Evaluation of Sensory Attributes of cowhide leather tanned using different chromium concentrations}

\subsubsection{Fullness}

Statistical differences regarding fullness were found in the cowhide leathers. According to the Kruskal-Wallis test, the highest values were obtained using $7 \%$ chromium in tanning (T3), given that results are equal to 4.80 points, an "excellent" rating as per Hidalgo (2017). Additionally, the lowest values were obtained by the hides tanned using low chromium concentrations, namely, 5\% (T1), considering that results are equal to 3.20 points.

\subsubsection{Fineness of grain}

Highly significant differences $(P<0.01)$ regarding fineness of grain were determined, as a result of tanning using different concentrations of chromium. Likewise, the highest results were obtained by the hides subjected to treatment T3 $(7 \%)$, achieving 4.60 points, an "excellent" rating as per Hidalgo (2017). Next were the results of hides subjected to treatment T2 (6\%), achieving 4.20 points, a "very good" rating according to Hidalgo (2017). Finally, the fineness of grain of hides subjected to treatment T1 (5\%) obtained the lowest results, achieving 2.80 points in means separation using the Tukey test (ANOVA).

\subsubsection{Roundness}

Highly significant differences $(P<0.01)$ regarding roundness were determined. Thus, the best results were found in the hides subjected to treatment T3 (7\%), achieving 4.80 points, an "excellent" rating as per Hidalgo (2017). Next, in descending order, roundness reported in the hides subjected to treatment T2 $(6 \%)$ achieved 4.20 points and a "very good" rating. In addition, leathers subjected to treatment T1 $(5 \%)$ reported the lowest results, achieving 3.0 points and a "good" rating.

\section{Second stage}

2.1. Evaluation of Physical Resistance of cowhide leathers tanned using $5 \%$ oxazolidine combined different tara concentrations $(12 \%$, $15 \%$ and $18 \%$ )

\subsubsection{Tensile strength}

No statistical differences $(P<0.05)$ regarding tensile strength were determined, as a consequence of tanning using different concentrations of Caelsalpinia spinoza (tara) combined with $5 \%$ oxazolidine. Thus, the highest values were found in the hides subjected to treatment T2 (15\%), producing results of $2855.15 \mathrm{~N} / \mathrm{cm}^{2}$; followed by the values of hides in treatment T3 (18\%), producing results of 2717.64 $\mathrm{N} / \mathrm{cm}^{2}$. In addition, hides subjected to treatment $\mathrm{T} 1$ $(12 \%)$ reported the lowest values, achieving tensile strength values of $2501.54 \mathrm{~N} / \mathrm{cm}^{2}$.

\subsubsection{Percentage of elongation}

No statistical differences $(P>0.05)$ regarding the percentage of elongation were determined in the analysis of variance between the means of treatments, as a result of tanning using different concentrations of tara combined with oxazolidine. Thus, the highest percentage of elongation was reported in treatment T3 $(18 \%)$, producing values of $61.54 \%$, which descended to $59.55 \%$ in hides subjected to treatment T1 $(12 \%)$. In addition, hides subjected to treatment T2 $(15 \%)$ reported the lowest percentage of elongation results, achieving values of $56.93 \%$.

\subsubsection{Tear strength}

No significant differences regarding tear strength were determined $(P>0.05)$ between the treatments, as a result of tanning using different concentrations of tara combined with oxazolidine. The highest results were found in treatment T3 $(18 \%)$, with 117.78 $\mathrm{N}$ (see Table 6), that is, greater tear strength of cowhide leathers was obtained using higher concentrations of tara combined with oxazolidine. In that respect, Vargas and Amurio (2017) state that

Table 5. Evaluation of Physical Properties of cowhide leather tanned using different chromium concentrations (5\%, $6 \%$ and $7 \%$ ). First stage.

\begin{tabular}{|c|c|c|c|c|c|c|c|c|c|c|}
\hline \multirow{3}{*}{$\begin{array}{l}\text { Physical characteristics } \\
\text { Tensile strength }\left(\mathrm{N} / \mathrm{cm}^{2}\right)\end{array}$} & \multicolumn{6}{|c|}{ Chromium concentrations } & \multirow{3}{*}{$\begin{array}{c}\text { SE } \\
129.46\end{array}$} & \multirow{3}{*}{$\begin{array}{c}\mathbf{P} \\
0.0142\end{array}$} & \multirow{3}{*}{$\begin{array}{l}\text { Sig. } \\
\text { NS }\end{array}$} & \multirow{3}{*}{$\begin{array}{l}\text { CV } \\
16.43\end{array}$} \\
\hline & \multicolumn{2}{|l|}{$\begin{array}{l}5 \% \\
\text { T1 } \\
\end{array}$} & \multicolumn{2}{|l|}{$\begin{array}{l}6 \% \\
\text { T2 } \\
\end{array}$} & \multicolumn{2}{|l|}{$\begin{array}{l}7 \% \\
\text { T3 } \\
\end{array}$} & & & & \\
\hline & 1341.12 & a & 1887.53 & a & 2057.37 & a & & & & \\
\hline Percentage of elongation (\%) & 56.90 & a & 80.50 & a & 94.38 & a & 6.78 & 0.0056 & $* *$ & 19.62 \\
\hline Tear strength $(\mathrm{N})$ & 54.35 & a & 53.67 & a & 55.85 & a & 1.32 & 0.157 & NS & 5.4 \\
\hline
\end{tabular}

Source: Prepared by the author. 
when no damage is produced on the leather surface nor in its internal structure after rubbing the leather pieces together or against foreign bodies, it is implied that it is a very rigid leather that even the slightest stretch will break. It is therefore determined that achieving greater resistance of leather to scratches caused by friction against its own surface or foreign bodies that weaken the interwoven fibers, provokes the appearance of cracks, leading to a premature aging of the leather (Casa Química Bayer, 2007).

\subsection{Evaluation of Sensory Attributes of cowhide} leather tanned using $\mathbf{5 \%}$ oxazolidine combined with different concentrations of tara $(12 \%, 15 \%$ and $18 \%$ )

\subsubsection{Fullness}

The Kruskal-Wallis test determined highly significant differences $(P<0.01)$ regarding fullness; the highest values were found in treatment T3 (18\%), obtaining 4.80 points and an "excellent" rating.

\subsubsection{Fineness of grain}

Highly significant differences $(P<0.01)$ regarding fineness of grain were determined, as a result of tanning using different concentrations of tara combined with 5\% oxazolidine. Thus, the highest values were obtained using $18 \%$ tara (T3), achieving val- ues of 4.70 points (see Table 7) and an "excellent" rating as per Hidalgo (2017).

\subsubsection{Roundness}

The Kruskal-Wallis test determined highly significant differences $(P<0.01)$ regarding roundness, as a result of tanning using different concentrations of tara combined with $5 \%$ oxazolidine. Thus, the highest results were obtained using $12 \%$ tara (T1), achieving 4.70 points and an "excellent" rating as per Hidalgo (2017). Next were the results obtained by the lot of hides tanned using $15 \%$ tara (T2), achieving 3.90 points and a "very good" scoring. In addition, the lowest results for roundness were obtained by the group tanned using $12 \%$ tara, achieving 3.40 points and a "good" rating.

\subsubsection{Suppleness}

Highly significant differences $(P<0.01)$ regarding suppleness of cowhide leather were obtained as a result of tanning using different concentrations of tara combined with oxazolidine. Leather suppleness improvement was observed when said material was exposed to lower concentrations of tara. The highest results were found in treatment T1 (12\%), achieving 4.50 points and an "excellent" scoring as per Hidalgo (2017); whereas, values descended in treatment T2 $(15 \%)$ to 3.60 points and a "very good" rating.

Table 6. Evaluation of physical resistance of cowhide leathers tanned using $5 \%$ oxazolidine combined with different concentrations of tara $(12 \%, 15 \%$ and $18 \%)$

\begin{tabular}{|c|c|c|c|c|c|c|c|}
\hline \multirow[t]{2}{*}{ Physical resistance } & \multicolumn{3}{|c|}{$\begin{array}{c}\text { Concentrations of tara combined with } 5 \% \\
\text { oxazolidine }\end{array}$} & \multirow[t]{2}{*}{ CV } & \multirow[t]{2}{*}{ SE } & \multirow[t]{2}{*}{ Sig. } & \multirow[t]{2}{*}{$\mathbf{P}$} \\
\hline & $12 \%$ & $15 \%$ & $18 \%$ & & & & \\
\hline Tensile strength $\left(\mathrm{N} / \mathrm{cm}^{2}\right)$ & $2501.54 \mathrm{a}$ & $2855.15 \mathrm{a}$ & $2717.64 \mathrm{a}$ & 25.52 & 217.21 & 0.52 & NS \\
\hline Percentage of elongation (\%) & $59.55 \mathrm{a}$ & 56.93 a & $61.54 \mathrm{a}$ & 12.58 & 2.36 & 0.40 & NS \\
\hline Tear strength $(\mathrm{N})$ & $101.23 \mathrm{a}$ & $101.36 \mathrm{a}$ & $117.78 \mathrm{a}$ & 19.24 & 6.50 & 0.14 & NS \\
\hline
\end{tabular}

Source: Prepared by the author.

Table 7. Values of sensory attributes of cowhide leathers tanned using $5 \%$ oxazolidine combined with different concentrations of tara $(12 \%, 15 \%$ and $18 \%)$.

\begin{tabular}{|c|c|c|c|c|c|c|c|}
\hline \multirow{2}{*}{ Sensory qualifications } & \multicolumn{3}{|c|}{$\begin{array}{c}\text { Concentrations of tara combined with } 5 \% \\
\text { oxazolidine }\end{array}$} & \multirow{2}{*}{ CV } & \multirow{2}{*}{ SE } & \multirow{2}{*}{ Sig. } & \multirow{2}{*}{$\mathbf{P}$} \\
\hline & $\begin{array}{c}12 \% \\
\mathrm{~T} 1\end{array}$ & $\begin{array}{c}15 \% \\
\mathrm{~T} 2\end{array}$ & $\begin{array}{c}18 \% \\
\mathrm{~T} 3\end{array}$ & & & & \\
\hline Fullness points & $3.10 \mathrm{c}$ & $3.80 \mathrm{~b}$ & $4.80 \mathrm{a}$ & 19.80 & 0.24 & 0.00 & ** \\
\hline Fineness of grain points & $3.50 \mathrm{c}$ & $4.10 \mathrm{~b}$ & $4.70 \mathrm{a}$ & 14.47 & 0.19 & 0.00 & ** \\
\hline Roundness points & $4.70 \mathrm{a}$ & $3.90 \mathrm{~b}$ & $3.40 \mathrm{~b}$ & 16.24 & 0.21 & 0.00 & ** \\
\hline Suppleness points & $4.50 \mathrm{a}$ & $3.60 \mathrm{~b}$ & $3.20 \mathrm{~b}$ & 14.90 & 0.18 & 0.00 & ** \\
\hline
\end{tabular}

Source: Prepared by the author. 


\section{Third stage}

3.1. Comparison of the physical properties of cowhide leathers tanned using $7 \%$ chromium versus tanning using $18 \%$ tara combined with $5 \%$ oxazolidine

\subsubsection{Tensile strength}

No statistical differences $(P>0.05)$ regarding tensile strength were determined upon comparing tanning using chrome versus tanning using different concentrations of tara combined with oxazolidine. Thus, the highest values were reported by tanning using tara (T2), obtaining values of $2214.49 \mathrm{~N} / \mathrm{cm}^{2}$, compared to chrome tanning (T1), which achieved values of $2057.37 \mathrm{~N} / \mathrm{cm}^{2}$. The typical error was 55.62 and 201.98 for treatments T1 (chromium) and T2 (tara), respectively. Mean tensile values equal to 2000.00 and $2177.07 \mathrm{~N} / \mathrm{cm}^{2}$ were registered and no repeated values were observed, indicating the absence of mode; however, standard deviation values were high, achieving values of $124.36 \mathrm{~N} / \mathrm{cm}^{2}$ and $451.63 \mathrm{~N} / \mathrm{cm}^{2}$.

\subsubsection{Percentage of elongation}

Highly significant differences $(P>0.05)$ regarding the percentage of elongation of cowhide leathers were determined. Thus, the highest results were reported by treatment T1 (tara), achieving values of $94.38 \%$ and a median of $80.50 \%$. In addition, leathers subjected to treatment T1 (chrome) registered values of $94.38 \%$ and $56.90 \%$. It should be noted that the values found in treatment T2 were $80.50 \%$.

\subsubsection{Tear strength}

Highly significant differences regarding tear strength of cowhide leathers were found. Thus, the highest results were reported by treatment T3 (tara), obtaining $117.78 \mathrm{~N}$ and a median of $101.36 \mathrm{~N}$; whereas the results obtained using chrome tanning were $55.85 \mathrm{~N}$ and a median of $54.35 \mathrm{~N}$. According to Chávez (2010), it is necessary to combine oxazolidine with vegetal tanning agents in order to achieve higher shrinking temperatures and obtain leather of a similar quality to those tanned using chrome.

\subsection{Evaluation of sensory attributes of cowhide leathers tanned using Chrome versus tanning using different concentrations of tara combined with $5 \%$ oxazolidine}

\subsubsection{Fullness}

Statistical differences $(P<0.05)$ regarding fullness values of cowhide leather were found. Thus, more effective results were found in leathers tanned using tara (T2), because the results obtained were 4.80 points, compared to leathers tanned using vegetal tanning agents, which obtained 3.80, and excellent and very good ratings as per Hidalgo (2017), respectively. According to Hoinacki (2009), this is corroborated by the negative effect of tanning with chromium salts, mainly because trivalent chromium is degraded into hexavalent chromium.

\subsubsection{Roundness}

No statistical differences regarding roundness of cowhide leather were reported as a result of the comparison of traditional tanning (chromium) with vegetable tanning (tara) methods. Thus, the highest values were found in treatment T2 (tara), achieving mean values of 4.80 points and excellent ratings, compared to chrome tanning that registered values of 3.60 points and very good ratings. In other words, results are similar in both tanning methods; however, as far as the environment is concerned, the best alternative is to work with tanning agents combined with oxazolidine due their lower pollution output (Córdova et al., 2013).

\subsubsection{Fineness of grain}

Statistical differences $(P>0.05)$ regarding fineness of grain of cowhide leathers were determined. Thus, mean values of 4.80 and 3.40 points were established for treatment T1 (chromium) and T2 (tara), respectively, as well as excellent and good ratings. In other words, the best results in fineness of grain are obtained using chrome tanning. In that regard, Cotance (2004) indicates that the fineness of grain is due to the combination of chrome and the carboxyl group of collagen, which forms a very strong bond that attracts the adjacent fiber, causing hair follicle closure and thus giving smoothness to the grain surface.

In addition, Bacardit (2005) mentions that tanning maintains the most desired properties of hide, namely, wear and humidity resistance as well as flexibility. Moreover, the variety of links formed between tanning agents and cross-linked collagen present in hides makes the leather external appearance pleasant to the touch and sight without changing its natural fibers structure; thus, enabling the availability of the material in different places regardless of weather conditions so as to satisfy consumer demand.

\section{Behavior of leather in the manufacture of leather goods}

\subsection{Stitching}

Sensory testing performed on leather goods made with leathers tanned using different concentrations 
of Caelsalpinia spinosa (tara) determined, among the mean highly significant differences, that the highest values were found in briefcases manufactured with leathers subjected to treatment T3 (18\%), with 4.80 points and excellent ratings as per the scale established by Santillan (2017). The values decreased in the items manufactured with leathers subjected to treatment T2 (15\%), which obtained 4.30 points and a very good rating. In contrast, the lowest values were found in the items manufactured with leathers subjected to treatment T1 (12\%), which obtained 3.60 points and a good rating. Also, Soler (2004) indicates that stitching consists on the assembly of leather pieces by means of operations such as sewing, gluing, accessories and metal fittings placement, etc., thus, to endure this stage of the industrial process, which is often intense, leather must have adequate strength in order to prevent creases and deformations that deteriorate the appearance of footwear.

\subsection{Cut}

Highly significant differences regarding the variable leather cut were determined as a result of tanning using different concentrations of Caelsalpinia spinose combined with $5 \%$ oxazolidine. Thus, the highest results were obtained using higher concentrations of tara (T3), considering that the judge determined values of 4.90 points and excellent scoring, which descended to 4.30 points and very good scoring. Whereas the lowest values were reported by treatment T1 (12\%), with 3.90 points and a good rating.

\section{RECOMENDATIONS}

- Tanning cowhides using oxazolidine and Caelsalpinia spinosa improves hide classification, reduces defects, enhances physical and sensory properties, and provides hides with high stability to endure manufacturing processes and time, eliminating the presence of chromium III.

- The concentration of oxazolidine (5\%) combined with $12 \%$ Caelsalpinia spinosa enhances the appearance and physical properties of cowhide leather. In addition, shrinkage temperature is reached at $80^{\circ} \mathrm{C}$, economizing the process, where the economic returns are greater and greatly exceed profits generated by tanning with chromium.

- The use of oxazolidine combined with Caelsalpinia spinosa as a tanning agent makes it possible to obtain quality leathers that can be used by leather goods businesses.
This is demonstrated by the physical and sensory testing results and, primarily, the manufactured goods rating.

- There is a small increase in the Biological Oxygen Demand (BOD) and Chemical Oxygen Demand (COD) as a result of tanning using oxazolidine combined with Caelsalpinia spinosa that does not comply with the regulations set in the Texto Unificado de Legislación Secundaria del Ministerio del Ambiente (TULSMA) [Unified Text of Secondary Legislation of the Ministry of the Environment]. It was possible to reduce this value to below the permissible limit when conducting the treatment.

- The evaluation of leather behavior under the most representative manufacturing conditions determined that the best results regarding leather stitching and cutting were obtained using $18 \%$ tara combined with $5 \%$ oxazolidine (T3), scoring 4.80 and 4.90 points, respectively, and excellent rating.

\section{CONCLUSIONS}

- Using a combination of Caelsalpinia spinosa (tara) with oxazolidine as an ecological substitute of chromium in the tanning process of cowhides complies with environmental regulations, as the use of chromium in tanning is banned due to the negative effects it has on the environment upon degrading into hexavalent chromium, even after the end-product has been discarded (Hoinacki, 2009).

- Use a combination of $12 \%$ Caelsalpinia spinosa (tara) and $5 \%$ oxazolidine to produce leathers that meet the quality standards in physical and sensory testing, which are recommended for the manufacture of various leather goods, as well as European Ecolable criteria, for leather.

- Conduct research the use of Caelsalpinia spinosa combined with oxazolidine in different types of hides, such as sheep and goat hides or of minor species such as birds, fish, rabbits, among others, to validate the new technology, addressing at the same time the need for raw material at a lower cost (Chávez, 2010).

\section{REFERENCES}

[1] Bacardit, A. (2005). Química técnica del cuero. Cataluña, Spain: COUSO. 
[2] Silvateam (n.d.). Cuero curtido al vegetal. Silvateam. Retrieved from https://www. silvateam.com/es/quienes-somos/extraidos-dela-naturaleza/cuero-curtido-al-vegetal.html

[3] Casa Química Bayer (2007). Resistencia al frote del acabado del cuero. Barcelona, Spain: IMANAL.

[4] Chávez, A. (2010). Descripción de la nocividad del cromo proveniente de la industria curtiembre y de las posibles formas de removerlo. Revista Ingenierías, 9(17), 41-49. Retrieved from https:// dialnet.unirioja.es/descarga/articulo/4845697.pdf

[5] Córdova, H., Vargas, R., Téllez, L., Cesare, M., Becker, R. \& Visitación, L. (2013). Influencia del uso de acomplejantes en el baño de curtido sobre la calidad final del cuero. Revista de la Sociedad Química, 79(4), 388-397.

[6] Cotance, A. (2004). Ciencia y Tecnología en la Industria del Cuero. Igualada, Spain: Curtidores Europeos.

[7] Hidalgo, L. (2017). Escala de calificación de las variables sensoriales del cuero bovino curtido con diferentes niveles de tara. Riobamba, Ecuador: ESPOCH.

[8] Hoinacki, E. (2009). Peles e couros. Origens, defeitos e industrialização. Porto Alegre, Brazil: SENAI/RS.
[9] INESCOP (2011). Piel Respetuosa con el Medio Ambiente Curtida con Oxazolidina. Alicante, España: INESCOP.

[10] Patudo, A. (2012). Qué es hipótesis y cómo se estructura. Retrieved from https://es.scribd. com/document/105144657/Que-es-hipotesisy-como-se-estructura

[11] Reissig, P. (2006). Innovación en Cuero = Oportunidad para el Diseño. Retrieved from https://www.inti.gob.ar/disenoindustrial/pdf/ reissig.pdf

[12] Mayta, R. \& Mayta, J. (2017). Remoción de cromo y demanda química de oxígeno de aguas residuales de curtiembre por electrocoagulación. Revista de la Sociedad Química, 83(3), 331-340.

[13] Santillán, A. (2017). Escala de apreciación de las características funcionales del cuero bovino. Riobamba, Ecuador: EI Al-CE.

[14] Soler, J. (2004). Procesos de curtido. Barcelona, Spain: CETI.

[15] Vargas, D. \& Amurrio, D. (2017). Alternativa de proceso de curtido con alto agotamiento de Cromo para las curtiembres tradicionales de la ciudad de Cochabamba. Acta Nova, 8(1), 3-30. 